Annuaire suisse de politique de développement

9 | 1990

Annuaire Suisse - Tiers Monde 1990

\title{
Flux internationaux de capitaux d'origine illicite - La Suisse face aux nouvelles stratégies
}

Paolo Bernasconi

\section{(2) OpenEdition}

Édition électronique

URL : http://journals.openedition.org/aspd/1322

ISSN : 1663-9669

\section{Éditeur}

Institut de hautes études internationales et du développement

Édition imprimée

Date de publication : 1 janvier 1990

Pagination : 199-229

ISSN : 1660-5934

Référence électronique

Paolo Bernasconi, «Flux internationaux de capitaux d'origine illicite - La Suisse face aux nouvelles stratégies ", Annuaire suisse de politique de développement [En ligne], 9| 1990, mis en ligne le 28 mars 2013, consulté le 07 septembre 2020. URL : http://journals.openedition.org/aspd/1322 


\title{
Flux internationaux de capitaux d'origine illicite La Suisse face aux nouvelles stratégies
}

\author{
Paolo Bernasconi*
}

\section{Politique criminelle et politique étrangère}

"Grâce à cette décision nous aurons finalement les moyens de combattre l'évasion fiscale."

Cette déclaration a été faite par M. Jacques Delors, président de la Commission de la Communauté Européenne, à l'issue de la publication de la décision de renforcer et de coordonner la coopération internationale contre le blanchiment d'argent, décision adoptée lors de la rencontre au sommet qui s'est tenue à Paris le 14 juillet 1989 entre les sept pays les plus industrialisés du monde (Groupe de travail du Sommet de l'Arche).

Les grands thèmes à l'ordre du jour de cette rencontre relevaient tous de la politique économique mondiale. II est du moins étonnant qu'une question de politique criminelle fasse l'objet de débats de telle envergure et cela pour la deuxième fois déjà. En effet, la même question a donné lieu à une première décision lors du sommet de Toronto en 1987.

* L'auteur, exerçant actuellement comme avocat à Lugano $(\mathrm{CH})$, est chargé de cours en droit pénal économique aux Universités de St-Gall et de Zurich. II a été magistrat responsable du Ministère Public de Lugano entre 1969 et 1985. II a rédigé le projet de loi pour la punissabilité du blanchiment d'argent du Ministère suisse de la Justice. 
II est vrai que la consommation croissante de stupéfiants dans toutes les régions du monde, dans les pays industrialisés comme dans le Tiers Monde, à l'Ouest ainsi qu'à l'Est, représente un problème d'ordre social et sanitaire majeur. Les conséquences en sont graves: les revenus prodigieux encaissés chaque année par les responsables de la production et du trafic dans le monde confèrent à leurs organisations un pouvoir qui constitue un véritable danger pour l'ordre public et le fonctionnement de l'organisation étatique dans de nombreux pays. Le cas de la Colombie en est un exemple frappant.

Mais le fait que $M$. Delors ait évoqué explicitement et en public la question de l'évasion fiscale en l'associant directement à la lutte contre le blanchissage d'argent, met en évidence la relation directe qui existe entre une stratégie de politique judiciaire et un souci majeur touchant aussi de près l'économie des pays industrialisés: l'évasion fiscale et la fuite des capitaux.

Voilà donc la politique criminelle qui devient partie intégrante de la politique économique internationale et, par conséquent, de la politique étrangère des Etats. En Suisse, le sujet est bien connu depuis des années; il suffit de se rappeler dans quelle mesure les relations entre la Suisse et les Etats-Unis ou certains pays de la Communauté européenne ont pu être troublées à cause du non-respect de l'entraide judiciaire en matière pénale et fiscale.

II est donc utile de rappeler dans ce contexte les fondements juridiques permettant de distinguer les différentes raisons pour lesquelles des capitaux se trouvent en fuite:

- capitaux d'origine criminelle constituant le revenu d'un crime ou d'un délit de droit commun;

- capitaux d'origine fiscale en fuite à l'étranger parce qu'ils ont été soustraits à l'imposition fiscale nationale;

- capitaux exportés d'un pays en violation des normes légales prévoyant des restrictions en matière de change et de devises.

La Suisse, confrontée à l'afflux de tels capitaux en provenance de ces trois sources, a toujours su, dans sa législation, sa jurisprudence et sa politique bancaire et financière, faire cette distinction fondamentale.

Compte tenu des nouvelles stratégies du crime organisé international d'une part, des nouvelles stratégies abordées par les organisations internationales, notamment sous l'influence persistante des autorités nord-américaines d'autre part, il est nécessaire d'approfondir l'étude des conséquences liées à cette distinction. Dans notre analyse, nous nous bornerons donc à l'étude des aspects du droit pénal et nous nous contenterons d'un renvoi bibliographique pour ce qui touche à l'aspect fiscal et économique. 


\section{Le blanchiment d'argent d'origine criminelle}

\section{Répartition géographique}

Sur le plan géographique, on peut diviser les territoires des différents pays selon la fonction que les organisations criminelles leur attribuent. En effet, il y a des territoires sur lesquels sont plutôt concentrées les activités criminelles. II y en a d'autres sur lesquels sont plutôt concentrées les activités de soutien telles que le blanchissage d'argent. L'on peut constater un flux de biens partant des pays de la première catégorie en direction des pays de la deuxième catégorie.

Prenons pour exemple la mafia italo-américaine: I'utilisation de la violence et de la corruption dans le but d'obtenir le contrôle de certaines zones géographiques ainsi que de certaines activités illicites se concentre sur les territoires des Etats-Unis et de l'Italie méridionale. Par contre, dans les pays choisis pour le blanchiment, la mafia s'abstient, en général, de commettre des infractions majeures. Comme tous schémas, celui-ci se révèle quelque peu sommaire et imprécis. Prenons comme autre exemple les activités liées à la drogue, qui constituent actuellement le revenu principal du "crime organisé". Selon le schéma établi, il existerait trois catégories de territoires: les pays producteurs, les pays de consommation et les pays de blanchiment. En réalité les pays producteurs, comme ceux du Sud-Est asiatique ou ceux d'Amérique latine, connaissent, à côté de la production, la consommation et le blanchiment des revenus. De même, dans les pays de consommation où est concentré le marché de la distribution des stupéfiants (Amérique du Nord et Europe occidentale), l'on retrouve des activités de blanchiment très importantes. C'est le cas de la place financière de Miami, considérée actuellement par les autorités des Etats-Unis comme le grand centre mondial de blanchiment.

En dépit de ses imprécisions, notre schéma permet une répartition du moins sommaire sur la carte géographique mondiale: il y a des pays où se concentrent les activités criminelles et il y a des pays où se concentrent essentiellement des activités de blanchiment.

La liste des territoires des pays ou régions intéressés par le blanchiment de leurs revenus peut être établie comme suit:

- le territoire des Etats-Unis - en raison d'une concentration importante des activités produisant des revenus illicites ;

- le Canada - du fait qu'aucune restriction n'est prévue à l'importation de devises et de biens des Etats-Unis, un contrôle douanier au sujet des origines de ces biens est pratiquement impossible ;

- les pays refuges fiscaux (Panama, les îles des Caraïbes, les îles anglaises de la Manche, le Liechtenstein, le Luxembourg, lîle de Malte, Singapour et Hong-Kong);

- et enfin les centres financiers d'importance internationale tels que Londres et la Suisse. Il est évident que, dans les pays dont le marché des devises est libre et dans lesquels se concentrent les mouvements de capitaux les plus 
importants du monde, il est beaucoup plus facile de parvenir à cacher des biens d'origine criminelle.

\section{Données statistiques}

Du point de vue quantitatif, le phénomène n'a pas encore été suffisamment évalué. En droit pénal cette difficulté est bien connue; le chiffre noir (dark number, c'est-à-dire la partie des crimes qui n'est pas découverte par les autorités) constitue un facteur typique dont il faut toujours tenir compte. A cela s'ajoute le fait qu'en matière de trafic illicite de stupéfiants, d'armes ou autre, la source première d'information, c'est-à-dire la partie lésée, fait défaut à l'autorité judiciaire. Dans ce type d'infraction, il n'existe pas de victime qui soit directement lésée. De plus, en matière de blanchiment d'argent, le chiffre noir est vraisemblablement plus élevé que pour d'autres infractions car le blanchiment est une infraction dont le but est précisément constitué par l'effacement de preuves et l'occultation du produit du crime. Par conséquent on ne dispose que de chiffres estimés concernant certains pays et qui sont loin de faire l'unanimité parmi les experts.

Force est de reconnaître également une certaine retenue de la part des autorités dans le rassemblement de ces données qui sont pourtant si nécessaires. Premier exemple: en 1980, le Conseil de l'Europe approuva une Recommandation No $R(80) 10$ relative aux "Mesures contre le transfert et la mise à l'abri des capitaux d'origine criminelle". La mise en pratique de cette Recommandation prévoyait l'établissement d'un premier rapport intermédiaire de la part des Etatsmembres pour 1985. Ce n'est qu'en 1987 que l'on a entamé cet examen. Deuxième exemple: dans le cadre des études préliminaires à la Recommandation susmentionnée, l'on recueillit en Suisse les données concernant l'importance des biens d'origine criminelle qui avaient été saisis par les autorités judiciaires helvétiques pendant la période 1968-1978 (26 cas ont été recensés dont la première affaire, d'un montant négligeable d'ailleurs, remontait à 1971). Le volume total des fonds d'origine criminelle saisis représentait une valeur de presque 100 millions de francs suisses. Malheureusement, cette statistique n'a pas encore été mise à jour, le Conseil fédéral ayant accepté une deuxième requête parlementaire en ce sens seulement en 1989 sous la pression politique due à l'éclatement de l'affaire Kopp.

II est probable qu'une statistique des affaires de blanchiment entre la période de 1979 à 1989 mettra à jour également une augmentation considérable du montant total des biens d'origine criminelle séquestrés en Suisse par les autorités judiciaires. II ne faut cependant pas nécessairement en déduire que le nombre de cas de blanchissage d'argent sur le territoire suisse a augmenté. En effet, d'autres facteurs ont probablement joué un rôle très important. En premier lieu, une amélioration substantielle de la collaboration entre les autorités judiciaires et de police a pu être constatée, notamment les demandes de coopération provenant de pays dans lesquels est concentrée la production de biens 
d'origine criminelle (Etats-Unis et Italie) qui ont permis aux autorités suisses de réunir les informations nécessaires pour démanteler les endroits de dépót de biens illégaux. En deuxième lieu, la découverte de cas qui ont suscité un écho important dans l'opinion publique a sensibilisé autant les autorités judiciaires que les milieux financiers.

\section{Les "générations" de blanchissage d'argent}

A défaut de données quantitatives, bornons-nous à une esquisse basée sur des cas significatifs. Un critère distinctif est celui de la nature de l'infraction dite "principale", c'est-à-dire l'infraction qui a produit les biens destinés à être blanchis. Au cours des deux dernières décennies, il est possible de dresser une liste de générations d'infractions principales. Notons que ces découvertes ont été possibles aussi grâce à la collaboration des instituts bancaires et financiers. En effet, les fonctionnaires de ces institutions ont été appelés à témoigner à la barre, permettant ainsi de rassembler la documentation qui fut utilisée comme pièces à conviction par les autorités judiciaires.

a) Dès le début des années 60, le "crime organisé" est devenu, avant tout aux Etats-Unis, un véritable "Etat dans l'Etat". Les organisations criminelles utilisent de plus en plus d'importants moyens financiers pour corrompre la police et d'autres autorités qui travaillent en vue d'entraver la poursuite de leurs activités criminelles. C'est surtout le trafic de papiers-valeurs volés ou falsifiés aux USA qui, dans les années 60, a atteint des proportions importantes. Ce trafic est devenu l'une des sources principales des revenus réalisés de façon illicite par le le crime organisé.

C'est ainsi qu'en 1963 déjà, les autorités américaines contactèrent pour la première fois la Suisse en vue de stipuler avec elle un Traité bilatéral d'entraide en matière pénale. Ce traité ne vit le jour que dix ans plus tard, le 25 mars 1973 exactement. II fut ratifié en 1975 et entra en vigueur en 1977. La durée des négociations a mis en évidence les difficultés de concilier les méthodes d'enquête américaines avec certaines limites touchant la sauvegarde du secret bancaire. II s'agissait du premier traité bilatéral en la matière pour les Etats-Unis et pour la Suisse. II servit de modèle pour une série de traités similaires stipulés par les Etats-Unis dans les années suivantes. L'une des grandes nouveautés était le fait d'avoir inscrit des dispositions prévoyant une coopération élargie en vue de fournir des moyens de preuve dans le cadre d'enquêtes américaines quant aux personnes liées au crime organisé (Art. 6 - Art. 8 du Traité).

Ce résultat était devenu possible notamment en raison de la constatation exprimée par le Conseil fédéral dans son Message concemant ce traité :

"Ce fut surtout le trafic de papiers-valeurs volés et falsifiés qui, à partir de 1966, a pris des proportions importantes en devenant l'une des sources principales de revenus réalisés par des moyens illicites(...) les investigations des autorités américaines permettent de conclure de manière irréfutable qu'une partie 
importante de ces transactions se déroulent dans d'autres pays(...) également en Suisse où des façons très sophistiquées pour les déguiser ont été adoptées".

Cette déclaration constituait en fait la reconnaissance, du moins partielle et indirecte, des accusations graves émises par Robert Morgenthau, State Attorney of Southern District of New York, au cours de sa déposition lors du hearing tenu le 9 décembre 1968 devant le Committee on banking and currency de la Chambre des représentants.

b) Au cours des années 70 une vague d'enlèvements de personnes déferla sur certains pays d'Europe. En Italie, plusieurs années de suite, l'on compta par année plus de 200 cas d'enlèvements de personnes. L'on estima qu'il ne s'agissait que de la moitié de tous les cas d'enlèvement car une partie des familles terrorisées par les auteurs des enlèvements ne signalaient pas la disparition de leur proche. Elles craignaient la stratégie "dure" des autorités judiciaires qui consistait à bloquer tous les biens de la famille afin de prévenir de nouveaux enlèvements de personnes. Les auteurs étaient organisés en bandes, des rôles et fonctions spécifiques étant répartis entre les différents membres de la bande en vue des enlèvements suivants. Une véritable masse de milliards de lires tombait entre les mains de ces groupes organisés. Une partie du blanchissage de cet argent se fit également en Suisse, comme le démontrèrent de nombreuses saisies ainsi que certains procès notamment dans la partie méridionale du canton du Tessin.

Certains enlèvements furent commis par des bandes terroristes. En 1975, un groupe de trois personnes appartenant à une organisation apparentée aux Brigades rouges fut arrêté à Lugano. Elles étaient en possession d'une somme d'argent importante provenant de la rançon payée pour la libération d'un ingénieur de Milan, qui a néanmoins trouvé la mort entre-temps. En 1977, à Chiasso, eut lieu l'arrestation de trois personnes appartenant à une organisation apparentée à la RAF ouest-allemande. Elles étaient en possession d'une partie de la rançon payée pour la libération d'un industriel enlevé à Vienne. Successivement, des rançons ont également été payées, à partir du territoire suisse, à I'IRA irlandaise ainsi qu'à une organisation japonaise.

Une première réaction importante à ces événements a été l'élaboration de la Recommandation No $R(80) 10$ relative aux "Mesures contre le transfert et la mise à l'abri des capitaux d'origine criminelle", adoptée par le Conseil des ministres du Conseil de l'Europe en 1980. Elle a été établie sur la base d'une étude à laquelle avaient notamment participé les experts suisses et italiens. Auparavant la Suisse pouvait déjà compter sur un instrument qui se révéla être, pour l'avenir, un instrument important pour la prévention du blanchissage d'argent.

II s'agit de la Convention relative à l'obligation de diligence des banques en cas d'acceptation de fonds et relative à l'usage du secret bancaire (CDB). Cette Convention est entrée en vigueur le 1er juillet 1977. II s'agit d'une Convention privée, entre la Banque nationale suisse d'une part et l'Association suisse des banquiers ainsi que toutes les banques suisses d'autre part. Elle constitua la 
réponse des milieux bancaires suisses à la découverte des malversations dans la gestion de la succursale du Crédit suisse de Chiasso, filiale d'une des trois grandes banques suisses. La Convention, renouvelée et améliorée le ler juillet 1982 et le ler juillet 1987, prévoit notamment l'obligation pour les banquiers d'identifier les clients ainsi que les ayants droit des fonds déposés en banque.

Pour ce qui est de la prévention et la répression des actes de terrorisme, une Convention internationale fut approuvée en 1977 à laquelle la Suisse a adhéré. Elle ne prévoit aucune disposition particulière en matière de blanchiment. En revanche, une telle disposition est prévue par la nouvelle loi anglaise en matière de terrorisme de 1988.

c) Ce fut surtout pendant les années 80 que l'on constata l'importance du blanchiment de biens provenant du trafic international des stupéfiants sur territoire suisse. Le cas le plus éclatant fut certainement celui connu sous le nom de "pizza connection". II s'agissait d'une organisation criminelle italo-américaine baptisée ainsi parce qu'une partie de l'argent recueilli par les dealers d'héroïne aux Etats-Unis était ramassée auprès de petites pizzerias. Le 9 avril 1984, une vaste opération préparée en collaboration entre les autorités américaines, italiennes, suisses et d'autres pays, permit l'arrestation simultanée d'une soixantaine de personnes dans ces différents pays. Lors de sa conférence de presse du 14 avril 1984, l'Attorney general américain déclara qu'il s'agissait du plus important succès dans l'histoire de la lutte menée contre la mafia par les autorités américaines. On estima que le trafic d'héroïne effectué par la "pizza connection" portait sur une valeur totale de 1,6 milliard de dollars. Toute une série d'autres cas avaient déjà été découverts, démontrant que les institutions financières suisses étaient exploitées non seulement par les organisations de trafiquants d'héroïne du Moyen et de l'Extrême-Orient, mais aussi par les organisations de trafiquants de cocaïne d'Amérique latine. Les difficultés des autorités judiciaires à obtenir la confiscation définitive des fonds saisis avaient conduit le Ministère public de Zurich à organiser une conférence de presse le 6 juin 1985 (Cf Tages Anzeiger du 24.8.1989).

Le procès de la "pizza connection", qui se déroula à Lugano en septembre 1985, mit en évidence les lacunes du droit pénal suisse en matière de punissabilité face au problème du blanchiment d'argent. L'année suivante, le Département fédéral de justice et police amorça les travaux préparatoires pour l'élaboration d'une nouvelle norme prévoyant la punissabilité du blanchissage d'argent dans le code pénal suisse (nouvel article 305 bis du code pénal). L'importance qu'accordent les autorités suisses et américaines à ce problème ressort également du Memorandum of understanding (MOU) sur l'entraide judiciaire en matière pénale, signé le 10 novembre 1987 entre le gouvernement de la Confédération suisse et le gouvernement des Etats-Unis d'Amérique. Ce Mémorandum n'est cependant qu'une déclaration commune d'intentions. II s'agit d'une simple description de la pratique adoptée par les autorités suisses et américaines pour améliorer leur coopération dans l'application des lois pénales sur le plan international. 
Mais c'est l'affaire de la Lebanon connection qui souleva une réaction inconnue jusqu'alors dans l'opinion publique et dans les milieux politiques et financiers suisses. La raison de cette réaction ne tient pas à l'importance des montants reconnus comme d'origine criminelle, mais au fait que cette affaire se transforma vite en scandale politique qui se termina par la démission forcée de Mme Elisabeth Kopp de son poste de chef du Département fédéral de justice et police. Cette démission fut la conséquence politique de la fonction exercée par son mari en tant que vice-président d'une société dont le nom avait été mis en relation avec des trafiquants de devises entre la Turquie et la Suisse. Dans son communiqué de presse, l'autorité judiciaire compétente avait précisé que le montant des devises transférées en contrebande depuis la Turquie pendant les deux dernières années atteignait deux milliards de francs suisses, dont une partie seulement était d'origine criminelle. La presse négligea immédiatement cette dernière précision et parle aujourd'hui encore de blanchiment de deux milliards de francs suisses d'origine criminelle. Même la Commission fédérale des banques enquêta et a rendu public ses résultats lors de sa conférence de presse annuelle en avril 1989. Entre autres, les déclarations suivantes furent faites (traduction libre de l'allemand):

"Le volume annuel des devises étrangères négociées en Suisse est de 80 à 100 milliards de francs, soit 5 à $8 \%$ du marché mondial. Ce marché est également utilisé par des organisations criminelles."

d) Le 16 novembre 1981, le juge Milton Pollack (ce n'est pas par hasard qu'il s'agit de la même juridiction que celle du State Attorney Morgenthau, c'est-àdire le Southern District of New York) prit des initiatives qui ne restèrent pas sans répercussions directes sur le droit pénal suisse. Il ordonna à une banque suisse de fournir au tribunal américain les données concernant une opération d'insider trading de l'un de ses clients; ces données concernaient des options sur des actions de la St. Joe Minerals Corporation. En cas de non-exécution, la banque suisse aurait été exclue des marchés financiers américains et aurait dû payer cinquante mille dollars de pénalité pour chaque jour de retard. Les sanctions dramatiques annoncées dans l'ordonnance ne furent pas mises à exécution parce que le client de la banque renonça à se prévaloir du secret bancaire. En automne de la même année, l'autorité de surveillance de la bourse américaine (Security and Exchange Commission, SEC) entama une procédure pour un cas d'insider trading concernant la reprise de la compagnie Santa Fé par la Koweit Petroleum Corporation. Cette nouvelle procédure visa également des banques suisses par le truchement desquelles des achats d'actions et d'options de la Compagnie Santa Fé avaient été effectués. Dans de nombreux cas, le recours à des institutions financières suisses pour effectuer des opérations d'insider trading a pu être prouvé.

La gravité de ces cas, notamment l'importance des intérêts économiques en jeu en raison de conflits juridictionnels, a suscité la réaction et des autorités politiques suisses et de l'Association suisse des banquiers. D'une part fut stipulé entre les gouvernements suisse et américain le Memorandum of understanding 
du 31 août 1982, d'autre part entrait en vigueur le 1er janvier 1983 la Convention XVI établie par l'Association suisse des banquiers concernant les opérations de insider trading effectuées aux Etats-Unis. Selon cette Convention, acceptée par la grande majorité des banques suisses, il était possible de mettre à disposition de la Security Exchange Commission américaine toutes les informations nécessaires pour ses enquêtes concernant l'insider trading, alors même que ce comportement ne faisait pas encore l'objet de sanctions en droit pénal suisse. Ce droit n'est entré en vigueur que lors de la promulgation du nouvel article 161 du code pénal suisse du 1er juillet 1988.

e) Ces dernières décennies sont également caractérisées par l'essor très important de la criminalité économique (white collars crimes). Dans ce domaine, certains cas de grande envergure pour lesquels il était nécessaire de trouver des refuges étaient en jeu. A titre d'exemple, il suffit ici de mentionner le cas Inving de 1971 qui suscita une réaction importante aux Etats-Unis, ou le cas de Licio Gelli, citoyen italien arrêté à Genève le 13 octobre 1982. En relation avec ce dernier cas, plus de cent millions de francs suisses censés provenir de la faillite du groupe bancaire Banco Ambrosiano de Milan furent bloqués dans une banque genevoise (voir bibliographie).

f) Les autorités judiciaires ont également découvert des biens cachés dans les banques suisses, produits de la corruption de fonctionnaires étrangers. II suffit de rappeler à ce sujet le cas du blanchiment des sommes provenant du scandalo dei petroli en Italie ou de celles provenant d'une corruption commise au Mexique (voir bibliographie).

g) En 1986, les avoirs déposés par l'ancien dictateur philippin Marcos ainsi que ceux déposés par l'ancien dictateur de Haïti, Duvalier, furent découverts auprès d'instituts financiers en Suisse. Ce problème n'était pas nouveau. Au printemps 1975, lors de l'effondrement du Vietnam du Sud et du Cambodge, les dirigeants de ces Etats avaient tenté d'envoyer une importante quantité d'or en Suisse. Le gouvernement helvétique promulgua alors, le 30 avril 1975, une Ordonnance interdisant l'entrée sur territoire suisse d'or provenant du Vietnam du Sud ou du Cambodge. La même année, en décembre, à l'occasion d'un débat parlementaire au sujet de l'acceptation de fonds déposés en Suisse par la famille de l'ancien chef d'Etat Hailé Sélassié, le chef du département fédéral des finances s'exclamait: "Mais comment pourriez-vous prétendre qu'un banquier suisse pose des questions à un chef d'Etat en charge concernant la légalité de l'acquisition de ses capitaux !". Suite aux expériences récentes, la position de la Commission fédérale des banques est la suivante:

"Les cas Marcos et Duvalier démontrent à quel point il peut être délicat pour des banques d'accepter en grande quantité des avoirs de chefs d'Etat étrangers. II appartient aux banques de régler clairement ce genre d'affaires par des directives appropriées." 
h) Des cas de blanchiment de produits de trafics d'autre nature, mais toujours pénalement punissables, ont été découverts sur le terrritoire Suisse. II s'agit en premier lieu du trafic d'armes illicite, c'est-à-dire du trafic d'armes non autorisé par les autorités du pays de production. Nous trouvons également les trafics de matériaux qui ont un caractère stratégique et dont l'exportation vers les pays de l'Est est interdite "matériaux Cocom". Finalement viennent s'ajouter les transactions financières qui sont liées aux activités clandestines des services secrets de différents Etats : le cas le plus connu est celui de l'Irangate découvert en 1987 (voir bibliographie).

i) Un dernier domaine, source de revenus illicites en quantité massive, est celui de la "traite" des femmes et des mineurs exercée à partir de certains pays du Tiers Monde en direction des pays de l'Europe occidentale et des Etats-Unis. En dépit des instruments mis en place par le droit international public qui sont destinés à assurer la coopération entre les Etats, aucun cas de blanchiment d'argent provenant de ce genre de trafic n'a encore été découvert en Suisse.

\section{La fulte de capitaux liée à des raisons fiscales}

\section{Catégories d'infractions fiscales}

Depuis toujours l'érosion des capitaux fiscalement imposables est une question très importante pour la fiscalité de tous les pays. Des estimations en la matière parlent de sommes colossales, qui sont soustraites au paiement des contributions dues, l'objectif étant la réalisation de ce que l'on appelle une "épargne d'impôts".

Une partie de ces sommes prend le chemin de l'étranger. On parle alors de fuite de capitaux pour des raisons fiscales. Mais dans cette catégorie des distinctions s'imposent :

a) L'épargne d'impôts peut être réalisée de façon absolument légale, c'est-àdire lorsque le contribuable essaie d'éviter qu'une activité soit reconnue imposable. L'on peut aboutir à ce résultat sans enfreindre aucune norme fiscale moyennant une analyse approfondie de la législation fiscale, nationale ou internationale. L'exemple type est celui du "Treaty shopping" où le contribuable, et c'est notamment le cas d'entreprises multinationales, choisit comme emplacement de ses activités ou de ses biens le territoire des pays qui offrent le maximum d'avantages fiscaux, soit de par leur législation nationale soit de par des traités qu'ils ont conclus avec d'autres pays (Tax Avoidance, évasion fiscale, elusione di imposta).

b) Le contribuable peut réaliser une épargne d'impôts en violant une norme fiscale lorsqu'il se soustrait totalement ou partiellement aux obligations prévues 
par la loi (Tax evasion, evasion fiscale, evasione fiscale). En droit suisse, on distingue deux grandes catégories d'évasion fiscale :

1. La soustraction fiscale, c'est-à-dire toute action ou omission tendant à soustraire à l'imposition, totalement ou partiellement, un élément imposable fiscalement.

2. La fraude fiscale, c'est-à-dire la soustraction fiscale commise en utilisant des documents falsifiés (documents comptables, bilans, etc.).

c) Une troisième catégorie, l'escroquerie en matière fiscale, réside dans la soustraction d'impót réalisée par toute manoeuvre frauduleuse. II s'agit d'une forme qui couvre l'utilisation de faux documents mais qui peut également être réalisée lorsque l'autorité publique a été induite en erreur par d'autres moyens astucieux (Abgabe- oder Steuerbetrug, fraude fiscale, truffa in materia tributaria, tax fraud).

A l'aide de la distinction susmentionnée nous trouvons trois conduites différentes à l'origine de la fuite de capitaux pour raisons fiscales. II peut y avoir: une conduite non punissable d'après la législation du pays d'origine et du pays destinataire; une conduite punissable d'après la législation du pays d'origine mais non punissable d'après la législation du pays destinataire ou vice versa et une conduite punissable dans les deux pays. Les capitaux tombant sous la troisième catégorie pourraient également être examinés en relation avec le chapitre précédent qui concerne les capitaux d'origine criminelle.

\section{Paradis fiscaux}

Les pays à fiscalité importante ainsi que certaines organisations internationales dressent depuis des années une liste des pays considérés comme refuges fiscaux (tax heavens, Steueroasen, paradis fiscaux, etc.). En général, tombent sous cette catégorie les pays qui connaissent d'une part un taux de fiscalité particulièrement bas ou des exemptions d'impôts et, d'autre part, les pays qui assurent des conditions fiscales privilégiées pour les contribuables domiciliés à l'étranger ou n'exerçant pas d'activité sur le territoire du pays concerné. Dans ces études, la Suisse y figure presque toujours. II est cependant très difficile d'établir le pourcentage des capitaux étrangers déposés en Suisse pour des raisons fiscales. Une casuistique très vaste a été publiée dans la littérature américaine publique ou privée.

\section{Casuistique suisse}

Les cas enregistrés par les autorités judiciaires suisses sont ceux qui ont fait l'objet de demandes d'entraide de la part d'autorités judiciaires étrangères sur la 
base du fameux article 3 de la Loi fédérale concernant l'entraide internationale en matière pénale (EIMP). Selon cet article, la Suisse exécute les demandes d'entraide présentées par des autorités étrangères dans le cadre de procédures pénales menées pour le chef d'escroquerie en matière fiscale. Mais l'examen de ces cas n'offre pas suffisamment d'éléments pour établir une statistique. D'abord parce que, à ce jour, certains Etats seulement se sont montrés intéressés à requérir la coopération suisse en application de la législation sur l'entraide internationale. II s'agit notamment de l'Allemagne, de la Suède, de l'Angleterre. D'autres Etats, notamment la France et l'Italie, n'ont presque jamais présenté de demandes d'entraide en matière fiscale. Ensuite, les cas publiés ne concernent pratiquement que des demandes d'entraide qui ont été acceptées. Elles sont au nombre de dix environ par année depuis l'entrée en vigueur de la nouvelle loi fédérale, c'est-à-dire dès le 1er janvier 1983. L'on constate qu'il s'agit en général de cas concernant des fraudes qui portent sur des sommes considérables. Le modus operandi est bien connu; il s'agit par exemple de concessions de prêts fictifs, d' opérations de sous-facturation ou sur-facturation, de l'utilisation de factures totalement fictives, etc.

\section{L'exportation Illicite de capltaux}

\section{Le marché libre des capitaux en Suisse}

La législation de plusieurs pays européens et hors d'Europe prévoit des restrictions à l'exportation de devises ou de capitaux en général. En cas de violation de ces restrictions, des sanctions pénales sont prévues.

En Suisse, le libre marché des capitaux est garanti. Aucune disposition ne restreint ni l'importation ni l'exportation de capitaux et de devises. Vouloir déterminer le pourcentage de capitaux étrangers en Suisse sortis de façon illégale du pays d'origine reviendrait à vouloir compter les grains de sable du désert. Des procédures pénales ont été engagées à l'étranger contre des citoyens non suisses accusés d'avoir exporté illégalement des capitaux ou d'avoir constitué des réserves de capitaux illégales à l'étranger. Nombre d'étrangers et de citoyens suisses - y compris quelques fonctionnaires de banque et fiduciaires- ont été accusés, voire même arrêtés à l'étranger, pour avoir organisé des réseaux de contrebande. Des fonctionnaires de douane étrangers ont même essayé de récolter sur territoire suisse des preuves concernant la constitution illicite de capitaux de ressortissants de leur nationalité. Mais ces cas ne sont absolument pas représentatifs.

Une fois de plus, ce fut grâce aux enquêtes des autorités judiciaires pénales suisses que l'on put se faire une idée, bien que très partielle, de l'ampleur et des mécanismes de ce trafic de capitaux par contrebande. Le premier cas fut celui des malversations découvertes dans la gestion de la filiale du Crédit suisse de Chiasso en 1977. Une grande partie de l'argent en question appartenait à des 
citoyens italiens résidant en Italie, argent qu'ils avaient fait parvenir en Suisse par le biais d'organisations de contrebande. C'est la raison pour laquelle les banques suisses se sont engagées, dans la Convention de diligence approuvée deux mois plus tard, en particulier "à ne prêter aucune assistance active dans le transfert de capitaux hors des pays dont la législation prévoit des restrictions en matière de placement de fonds à l'étranger".

En novembre 1988, l'affaire de la Lebanon connection révèle l'existence d'un réseau d'organisations de contrebande de devises en direction de la Suisse. II s'agissait d'argent provenant de la Turquie et du Moyen-Orient. A nouveau, ce ne fut que par réaction que l'on prit des mesures: la Commission fédérale des banques annonça, lors de sa conférence de presse annuelle, en avril 1989, l'élaboration de directives en vue d'une réglementation, voire d'une réduction du commerce de devises. Les milieux financiers et politiques ont réagi face à l'opinion publique, secouée par la publication des résultats des enquêtes, comme si le phénomène constaté avait été une véritable découverte. En réalité, l'importance de la fuite de capitaux par le canal de la contrebande n'a pas varié depuis des années et ce procédé est bien connu par les milieux financiers.

Une autre constatation apparaît régulièrement dans la presque totalité des cas de condamnation pour escroquerie au préjudice des investisseurs. L'on constate que la grande partie des investisseurs sont de nationalité étrangère et résidents à l'étranger. Il est notoire dans les milieux judiciaires et financiers que la grande majorité de ces victimes renoncent alors à porter plainte. Elles préfèrent la perte totale du capital investi au risque d'être dénoncées par les auteurs de l'escroquerie aux autorités compétentes dans leur pays pour la poursuite des infractions à la législation fiscale et de change.

\section{Recoupements entre infraction penale et Infraction fiscale}

\section{Un refrain}

L'on constate d'abord un certain nombre de faits concordants dans la littérature publiée par les autorités et celle qui l'est par les privés. Malheureusement, la production d'écrits en la matière est très vaste et se présente sous forme d'une palette hétérogène composée de reportages de presse, livres à sensation et publications scientifiques. Des études de cas concrets mélangent, indifféremment, crimes et infractions de droit fiscal ou de droit de change. Deux exemples sont significatifs : la liste de 128 cas établie en 1983 par un sous-comité du Sénat américain donne un inventaire d'infractions allant du trafic de drogue à la fraude fiscale jusqu'à l'utilisation abusive du secret bancaire. II en est de même avec le livre Dirty Money, qui provoqua un écho considérable aux EtatsUnis notamment parce qu'il avait été écrit par deux anciens procureurs publics. Le sous-titre de cet ouvrage : Swiss Banks, the Mafia, Money Laundering and White Collar Crime se passe de tout commentaire. 
Dans le cadre de l'OCDE, le comité des affaires fiscales publia le 3 juillet 1985 un rapport intitulé: "Fiscalité et usage abusif du secret bancaire" dont la conclusion contenait la proposition suivante: "Le Comité voit deux moyens d'avancer concrètement dans ce domaine:

- accroître les éléments d'information disponibles au plan interne, là où cela est nécessaire, en assouplissant les règles relatives au secret bancaire à l'égard des autorités fiscales,

- faire un plus large usage, grâce aux procédures d'échange de renseignements, des données pouvant être obtenues des banques."

De plus en plus les publications, comme les rapports de soutien aux mesures proposées, mélangent sans aucune distinction le domaine fiscal au domaine pénal. Voici un extrait de la préface de l'étude du sous-comité du Sénat américain: "Bankers, lawyers, offshore politicians and gangsters use offshore banking facilities as part of organized criminal enterprise."

Ce lien apparait encore plus nettement dans le rapport de l'OCDE: "Enfin, comme on le sait, le secret bancaire sert aussi à dissimuler des fonds provenant d'activités illégales (trafic de drogue et de biens volés) ou à échapper au contrôle des changes. II est donc prêté de plus en plus attention aux relations qui peuvent exister entre le crime organisé et le secret des affaires."

Une indication analogue figure dans la Résolution adoptée en 1978 par l'assemblée parlementaire du Conseil de l'Europe (Recommandation 833 (1978)) :"Constatant que les règles indûment restrictives sur le secret bancaire encouragent les infractions fiscales internationales (...), recommande au Comité des ministres: d'exorter les gouvernements des Etats-membres du Conseil de l'Europe à abolir les règles indûment restrictives sur le secret bancaire chaque fois que cela est nécessaire pour faciliter les recherches en cas de fraude fiscale ou de dissimulation de fonds provenant d'autres activités délictueuses, la protection de la vie privée restant garantie."

\section{Le rôle de la contrebande internationale de devises}

Il est évident que les revenus constituant le produit de crimes (trafic de drogue, white collar crimes, etc.) engendrent naturellement des infractions aux normes fiscales, puisque leurs auteurs doivent en cacher l'origine à toute autorité étatique. Lorsque ces crimes sont commis dans des pays où des restrictions au mouvement des capitaux sont en vigueur, le transfert du produit desdits crimes signifie nécessairement la violation de ces normes. Pour ce qui est de la Suisse, l'on constate que pratiquement tous les crimes à caractère international sont aussi en même temps liés à une violation de normes fiscales et de droit de change du pays où l'infraction principale a été commise.

Dans le but de cacher la masse croissante des revenus illégaux, les blanchisseurs d'argent recourent de plus en plus aux instruments classiques qui as- 
surent l'anonymat dans le secteur financier. II s'agit d'une part du secret bancaire, d'autre part du bénéfice des facilités accordées par les paradis fiscaux. Le Money launderer tente par conséquent de s'insérer dans le flux international des capitaux d'origines licites pour bénéficier des garanties ordinaires qui sont assurées à la clientèle honne̊te. En effet, dans la pratique judiciaire on constate que presque dans tous les cas importants de blanchissage d'argent et de criminalité économique l'on se trouve confronté à un usage abusif de ces garanties, notamment celles représentées par l'utilisation des sociétés implantées dans des pays refuges fiscaux (le Panama, les pays des Caraïbes, le Liechtenstein et les îles anglaises de la Manche (Jersey et Guernesey).

Les blanchisseurs d'argent ont également appris à recourir aux réseaux de la contrebande de devises fonctionnant depuis des décennies pour assurer la fuite des capitaux en direction des pays refuges. En Suisse, l'on a pu constater que ces réseaux avaient été utilisés pour l'exportation clandestine hors du territoire italien de rançons payées pour la libération de personnes enlevées ainsi que des biens obtenus dans d'autres pays par le trafic de drogue. Le rôle des réseaux de la contrebande de devises provenant de la Turquie en faveur des organisations criminelles a été démontré dans l'affaire Lebanon connection découverte en 1988. Les réseaux et les techniques employés pour transférer clandestinement de l'argent depuis les pays d'Amérique latine sont ceux aussi utilisés pour le transfert des produits du trafic de la cocaïne.

Ces mécanismes de contrebande constituent un abri très profitable pour le blanchisseur d'argent qui n'a pas besoin de constituer son propre mécanisme de blanchissage. En effet, comme il s'agit de transfert d'argent d'origine généralement non criminelle mais aussi de transfert d'argent en violation de normes fiscales et de droit de change, le blanchisseur peut se fondre dans la foule des clients des instituts financiers qui n'ont absolument rien à voir avec le crime. Cette constatation est valable pour le mécanisme le plus traditionnel, à savoir le transfert physique de l'argent d'un pays à l'autre, comme pour des mécanismes plus sophistiqués tels que la compensation ou des prêts fictifs.

En effet, les comptes bancaires utilisés pour assurer le transfert clandestin de capitaux entre pays d'origine et pays-refuge font état de mouvements d'importance extraordinaire. Par conséquent, il est très difficile de détecter parmi cette masse de transactions celles qui ont trait à des fonds d'origine criminelle. Même les personnes chargées de la gestion effective de ces comptes bancaires (appelés Sammelkonti) ne sont souvent pas en mesure de fournir des précisions aux autorités judiciaires qui les interpellent en qualité de témoin. Généralement, elles ne connaissent que leur correspondant à l'étranger, qui ne connaît à son tour que l'intermédiaire ou un courrier. A l'intérieur d'un tel réseau, l'on remarque que plus on s'approche des personnes qui ont un contact direct avec un client cherchant à exporter clandestinement de l'argent, moins on fournit d'éléments utiles à l'identification des personnes impliquées dans la contrebande. Ces personnes ne sont souvent connues que de leur surnom ou s'identifient moyennant un mot de passe.

Par conséquent, l'époque du blanchiment d'argent rapproche deux mondes 
traditionnellement séparés : l'organised crime et le white collars crime (Finanzunterwelt), c'est-à-dire la partie corruptrice et la partie corruptible du système financier et bancaire intemational. Ce rapprochement s'est fait par la nécessité de blanchir les produits du crime. II s'agit là d'une activité que les membres des organisations criminelles ne connaissaient pas encore suffisamment bien pour pouvoir la gérer et la contrôler eux-mêmes. Ainsi, la distinction des buts et des instruments existant traditionnellement entre le crime organisé et la criminalité des affaires est devenue toujours moins nette. La séparation très claire qui existait jadis entre le crime organisé et la violation de normes du droit fiscal et du droit des changes tend à disparaitre. L'utilisation d'instruments techniques identiques pour atteindre des buts aussi différents - criminels ou fiscals a pour effet que même des personnes n'ayant absolument aucune relation avec le crime organisé peuvent soudainement être impliquées - sans avoir aucune responsabilité pénale - dans une enquête engagée par les autorités judiciaires pénales.

\section{Le rôle des paradis fiscaux}

\section{a) Le refuge fiscal}

II existe une vaste littérature concernant les pays qui sont considérés comme des paradis fiscaux ou des refuges fiscaux. En effet, "le recours aux paradis fiscaux par diverses catégories de contribuables (personnes physiques aussi bien qu'entreprises) n'a cessé de progresser au cours des dernières décennies avec des résultats prejudiciables tant en termes de pertes de recettes qu'au regard de la moralité fiscale." (Voir l'étude de l'OCDE susmentionnée). En général, les paradis fiscaux classiques se caractérisent principalement par des taux d'imposition relativement faibles, un secret bancaire ou commercial d'un niveau élevé, l'absence de contrôle des changes, un secteur financier disproportionné par rapport à l'économie du pays concerné. Certains pays membres de l'OCDE ont établi des listes de pays considérés comme paradis fiscaux et des dispositions particulières s'y appliquent. Dans une étude des autorités américaines, la liste des pays suivants a été examinée : Antigua, Bahamas, Bermuda, Montserrat, Panama, Suisse, Caïman Islands. Y sont également mentionnés: les Antilles néerlandaises, Bahrein, Hong-Kong, Luxembourg, Channel Islands, Liechtenstein, St-Vincent et le Liberia.

\section{b) Le refuge pénal}

En réalité, ces listes ne sont guère utiles car n'y figure pas la distinction fondamentale suivante: les pays qui jouent le rôle de refuge fiscal et ceux qui jouent, en plus, le rôle de refuge pénal. Aussi difficile qu'il soit de trouver une définition exacte du refuge fiscal, on peut néanmoins se référer au concept législatif d'un 
pays qui cherche à attirer, par la concession d'avantages fiscaux, des revenus provenant d'activités exercées à l'extérieur de son territoire. De plus, le refuge pénal se caractérise par une législation ou une organisation de l'Etat qui exerce une attraction objective importante sur des personnes qui veulent commettre des crimes ou cherchent à échapper à une poursuite judiciaire. Les critères permettant de déterminer si un pays constitue un refuge pénal ou non sont les suivants :

\section{A. La législation commerciale et financière}

- Les conditions pour la constitution de sociétés commerciales, notamment le montant minimum du capital social à verser.

- Les dispositions concernant la responsabilité des administrateurs de sociétés commerciale.

- Les obligations régissant la tenue de la comptabilité et des bilans ainsi que leur remise aux autorités fiscales.

- Les dispositions concernant l'inscription dans les registres publics des noms des administrateurs et des gérants véritables des sociétés commerciales.

- L'obligation de soumettre les comptes annuels à une société de révision externe et indépendante.

- Le régime d'autorisation et de surveillance à l'égard des sociétés qui exercent une activité bancaire, parabancaire ou financière.

B. Les normes de droit pénal

- La responsabilité pénale des administrateurs de sociétés commerciales dans le cas des infractions commises dans l'exercice de l'activité de la société.

- La punissabilité du blanchissage d'argent.

- La punissabilité de l'Insider trading.

- L'obligation du banquier de venir témoigner en justice et de fournir des pièces à l'autorité judiciaire pénale.

- Les possibilités pour une autorité judiciaire de pouvoir séquestrer des avoirs d'origine criminelle déposés auprès de banques ou d'institutions financières.

- La punissabilité de l'escroquerie en matière fiscale.

- La punissabilité de la corruption de fonctionnaires étrangers.

- La faculté ou le devoir pour une autorité judiciaire nationale de fournir une entraide en matière pénale aux autorités judiciaires étrangères même lorsque l'enquête porte sur des questions couvertes par le secret bancaire et des affaires.

- La faculté ou le devoir pour une autorité judiciaire de fournir des informations et de la documentation aux autorités judiciaires étrangères concernant l'activité économique de tierces personnes qui ne sont pas accusées dans une procédure pénale étrangère. 
- L'obligation pour les banques d'identifier la clientèle ainsi que les ayants droit économiques des avoirs déposés en banque.

- L'obligation pour les banques de vérifier l'origine des fonds déposés.

- La faculté ou devoir de l'autorité nationale de concéder l'extradition pour escroquerie en matière fiscale.

L'application de ces différents critères peut servir à déterminer si un pays appartient ou non à la catégorie du refuge pénal. Naturellement, il ne faut pas se limiter à un examen purement comparatif, il faut également tenir compte du degré d'efficacité réel des normes existantes. II n'est par exemple pas possible de mettre sur le même plan deux pays dont les banques se sont donné un code de conduite bancaire en matière d'identification de la clientèle, si l'on constate que dans l'un de ces pays il existe une autorité de surveillance qui prononce un certain nombre de jugements tandis que dans l'autre pays aucun jugement n'a encore été émis des années après l'entrée en vigueur du code de conduite.

Un autre critère très important est celui du degré de l'organisation et du fonctionnement des structures administratives et judiciaires d'une part et celui des structures du secteur bancaire et financier de l'autre. II est évident que l'on ne peut pas mettre sur le même plan deux pays ayant la même législation mais dont l'un possède un système administratif surchargé, fonctionnant mal, voire même corrompu.

\section{c) Casuistique}

L'analyse proposée exige des connaissances approfondies du pays concerné. Le cadre de cette étude ne permet pas d'élaborer une liste des pays qui, en raison de leur législation ou de leur organisation, ne sauraient être qualifiés de refuge pénal. Nous ne pouvons que proposer quelques points de repère, étant parfaitement conscients du caractère sommaire de cette approche. Doit être considéré pays de refuge pénal par excellence le pays qui obtient un maximum de réponses négatives aux critères susmentionnés. Les refuges fiscaux des $\mathrm{Ca}$ raïbes et de l'Amérique centrale semblent correspondre d'assez près aux critères d'un refuge pénal contrairement aux refuges fiscaux de l'Europe occidentale. Cependant, l'on peut constater que les pays qualifiés de refuges fiscaux ont une nette tendance à revoir leur législation et leur organisation administrative afin de prévenir l'exploitation de leurs institutions par des criminels.

En conclusion, l'on peut dire que, depuis de nombreuses années déjà, la Suisse répond dans une large mesure positivement aux critères susmentionnés. Le Luxembourg et le Liechtenstein ont annoncé au printemps 1989 qu'ils allaient introduire la punissabilité du blanchiment d'argent dans leurs législations. Le Luxembourg a également annoncé un avant-projet prévoyant la punissabilité de l'Insider trading mais, par la même occasion, il a introduit, par une procédure d'urgence, des normes renforçant le secret bancaire. Le Liechtenstein de son côté a mené au but en 1989 une révision de la Convention de diligence ban- 
caire qui n'avait pas été adaptée à l'occasion des deux révisions de la Convention analogue exitante en Suisse. L'Autriche a pris des mesures pour renforcer le secret bancaire qui est réglementé par une loi pareille à celle adoptée en Suisse: en mars 1989, on a jugé opportun de reconnaître que ces mesures trouvent leur fondement dans la Constitution autrichienne.

\section{d) La position de la Suisse}

\section{L'entraide judiciaire internationale en matière pénale et en matière fiscale}

En examinant rétrospectivement la politique législative des pays considérés comme des refuges fiscaux, l'on constate que leur attitude se fonde essentiellement sur une distinction entre les comportements punissables selon le droit pénal ordinaire et les comportements tombant uniquement sous le coup de la législation fiscale ou de change. En ce qui concerne les premières infractions, on s'efforce d'assurer une large coopération, même dans le cadre des enquêtes conduites par des autorités étrangères. Quant aux autres, toute coopération est refusée, à l'exception des infractions fiscales qui sont également punissables pénalement dans le pays concerné. C'est précisément le cas de la Suisse. Sa politique en matière d'entraide internationale se caractérise traditionnellement par un refus absolu à toute coopération en matière fiscale, sauf pour ce qui est de la coopération dans le cadre des conventions internationales tendant à éviter la double imposition. Deux seules exceptions à cette attitude de principe ont été faites. Elles sont en faveur des Etats-Unis et personne ne s'en étonne: la première figure dans la Convention de double-imposition de 1951 et la deuxième dans le Traité d'entraide en matière judiciaire de 1973. Ce n'est que depuis l'entrée en vigueur le 1er janvier 1983 de la Loi fédérale sur l'entraide internationale en matière pénale que la Suisse accepte d'accorder l'entraide - à chaque pays également dans les cas où la procédure étrangère a pour but de poursuivre une escroquerie en matière fiscale. En revanche, cette exception n'a pas encore été prévue par les pays qui ont une législation semblable à la Suisse, comme le Luxembourg, le Liechtenstein et l'Autriche. En automne 1984, le Parlement suisse eut l'occasion de manifester sa volonté de ne pas poursuivre dans cette direction : il refusa la proposition du gouvernement de ratifier un accord international par lequel la Suisse se serait engagée obligatoirement à fournir des informations en matière fiscale aux autorités judiciaires des pays membres du Conseil de l'Europe.

\section{Secret bancaire et entraide judiciaire internationale}

La distinction entre infraction pénale et infraction de droit administratif, c'est-àdire de droit fiscal ou de droit de change, est fondamentale pour la question de la levée du secret bancaire. II est notoire que, selon la législation et la pratique 
suisses, le banquier ne peut pas opposer le secret bancaire lorsqu'il est appelé par l'autorité judiciaire pénale à témoigner ou à produire des documents bancaires. Mais ce principe n'est valable que lorsqu'il s'agit d'une procédure pénale avec témoignages ou production de pièces à conviction. En revanche, dans le cadre de procédures de caractère administratif, notamment les procédures fiscales, le secret bancaire ne peut pas être levé. Le Tribunal fédéral a eu maintes fois l'occasion de se prononcer à cet égard. Au sujet de la levée du secret bancaire dans le cadre de la poursuite d'une infraction punissable pénalement, il a établi le principe suivant :

"La législation sur l'entraide et les accords internationaux y relatifs doit contribuer à ce que la Suisse perde la réputation de pays où, gráce à la protection du secret bancaire, peuvent être mis à l'abri des biens patrimoniaux acquis de manière illicite." (Jugement du 10.9.1986 cons. 25). En effet, la législation et la pratique actuelles en Suisse assurent la réalisation de ce principe.

En matière de coopération fiscale, le Tribunal fédéral a établi le principe suivant :

"Il est exact que la Suisse n'accorde en principe aucune assistance judiciaire en matière fiscale. Ceci est en relation avec le secret bancaire garanti par l'article 47 de la Loi fédérale sur les banques, qui a joué un rôle important pour le développement de l'économie suisse."

Cependant, dans les cas où la Suisse accorde - en faisant exception au principe - une entraide en matière d'escroquerie fiscale, la saisie des biens soustraits au fisc étranger par une fraude fiscale n'est pas possible.

De toute manière les demandes d'entraide pour escroquerie en matière fiscale sont examinées rigoureusement afin d'éviter que l'autorité judiciaire étrangère requérante obtienne, sous la couverture d'une prétendue escroquerie en matière fiscale, des éléments de preuve qui en réalité doivent permettre la poursuite d'infractions fiscales pour lesquelles la Suisse n'accorde aucune entraide.

Un exemple significatif démontrant l'importance qu'accorde la Suisse à ces principes est constitué par le cas de l'enquête ouverte aux Etats-Unis contre le citoyen suisse Marc Rich et les organes des sociétés de son groupe pétrolier. Une des sociétés était domiciliée dans le canton de Zoug. La société Marc Rich AG à Zoug était accusée de fraude fiscale par l'autorité américaine notamment pour avoir soustrait au fisc américain des impôts d'un montant de $\mathbf{4 8}$ millions de dollars au cours des années 1980 et 1981. Le 22 juin 1983, le juge de New York ordonna à la société la remise de documents constituant des moyens de preuve sous peine de $\mathbf{5 0 . 0 0 0}$ dollars d'amende pour chaque jour de retard. Pour empêcher la remise de cette documentation, les autorités fédérales suisses ordonnèrent, le 12 août 1983, la saisie des dossiers requis par les autorités américaines. II s'agissait en effet d'une question de principe pour les autorités suisses : il fallait à tout prix éviter que les autorités américaines puissent obtenir, par une application extra-territoriale de leur droit, des moyens de preuve couverts par le secret situés sur territoire suisse sans avoir à se conformer aux conditions et à la procédure prévues par le Traité d'entraide judiciaire entre la Suisse et les Etats-Unis. 


\section{v. Convergence des Initiatives internationales et nationales contre la fulte des capltaux d'origine criminelle}

Nous n'entendons pas exposer ici la stratégie globale des différents pays et des organisations internationales face à la fuite des capitaux. Nous nous bornons aux mesures concernant les flux de capitaux d'origine criminelle. Au sujet des mesures destinées à combattre la fuite des capitaux pour des raisons fiscales ou de violation de restrictions nationales de droit de change, nous nous référons aux ouvrages mentionnés dans notre bibliographie. En effet, ce qui nous intéresse dans le cadre de cette étude, n'est pas tellement de présenter la panoplie complète des mesures, mais de démontrer qu'il existe une certaine convergence de ces mesures.

Initiatives contre la mise à l'abri de capitaux d'origine criminelle

\section{a) Initiatives à caractère pénal}

Au cours des dernières années, la prévention et la répresssion du blanchiment d'argent d'origine criminelle ont été à l'ordre du jour de plusieurs organes et comités d'étude, institutionnalisés ou non, de différentes organisations internationales. La dernière initiative a été celle des Nations Unies grâce à laquelle une Conférence diplomatique internationale s'est réunie à Vienne et a adopté le 20 décembre 1988 une Convention internationale contre le trafic des stupéfiants. Une centaine de pays adhérant à la Convention - y compris la Suisse - s'engagèrent notamment à introduire dans leur législation nationale la punissabilité du blanchiment d'argent et la confiscation du produit direct et indirect du crime.

Tout en reconnaissant l'importance de cette initiative, il faut pourtant en souligner les limites. La Convention ne porte que sur le blanchiment et la confiscation du revenu du trafic de stupéfiants. Alors que certains pays ${ }^{1}$ suivent cette ligne minimaliste, d'autres pays ${ }^{2}$ ont été plus loin en approuvant des dispositions pénales qui visent le revenu provenant d'autres crimes. II faut reconnaître que, notamment ces dernières années, les revenus du trafic de stupéfiants constituent la plus importante source financière du crime organisé. Mais il serait dangereux d'oublier les autres sources, trafic illicite d'armes, traite de femmes et d'enfants liée à l'exploitation sur le plan international de la prostitution, trafic de titres et papiers-valeurs volés ou falsifiés, trafic d'objets de marques falsifiés,

1) Cf. les lois récentes de France, Luxembourg, Belgique, Hong Kong, Singapour, Angleterre, Canada, Australie, Panama et Bahamas ainsi que le projet de la République Fédérale d'Allemagne.

2) Cf. les lois récentes des USA et de l'Italie (voir bibliographie sous III) ainsi que les projets gouvernementaux de la Suisse (du 12.6.1989) et du Liechtenstein. 
trafic d'argent falsifié, etc. ainsi que les revenus provenant de la banqueroute de groupes industriels ou bancaires et de la corruption.

Le gouvernement suisse a heureusement suivi la deuxième solution: son projet de loi, publié le 12 juin 1989, concernant la punissabilité du blanchiment d'argent vise les revenus de toute catégorie de crime.

Outre la punissabilité du blanchissage d'argent, la conférence de Vienne estime qu'il est indispensable d'adopter des mesures législatives pour renforcer la confiscation au niveau national comme au niveau international. II s'agit notamment d'assurer la confiscation lorsque le produit du crime a déjà été blanchi une ou plusieurs fois même s'il a déjà été réinvesti par une banque. On tend aussi à prévoir le renversement du fardeau de la preuve à l'égard du possesseur d'un bien dont la valeur patrimoniale dépasse manifestement les moyens financiers dont il dispose. En Suisse, un groupe d'étude du Département fédéral de Justice a été chargé d'étudier la révision des dispositions en vigueur (art. 58 du code pénal suisse).

\section{b) Initiatives à caractère bancaire}

Le Conseil des ministres du Conseil de l'Europe avait adopté déjà en 1980 une Recommandation prévoyant pour le système bancaire des pays membres l'obligation d'identifier la clientèle, ainsi que des mesures restrictives pour la mise à disposition de coffres de sécurité. Malheureusement ces Recommandations ne furent suivies que dans quelques pays et seulement partiellement . Le groupe des Dix, lors de sa réunion du 12 décembre 1988 à Bâle, a approuvé un code de conduite bancaire destiné non seulement aux membres de ce groupe, mais aux autres pays du monde. Ce code reprend les principes contenus dans la Convention de diligence signée par les banques suisses le 1er juillet 1977. Dans son projet concernant le blanchiment d'argent, le gouvernement suisse va jusqu'à proposer de sanctionner l'opérateur financier si celui-ci ne fait pas preuve de la diligence commandée par les circonstances lors de l'identification de sa clientèle ou des ayant droit économiques (art. 30s ter du Code Pénal). Si l'initiative remarquable du groupe des Dix commence à porter ses fruits, il apparaît que l'efficacité n'en sera garantie que lorsque le code bancaire adopté ne s'appliquera pas au secteur bancaire exclusivement, mais à tous les opérateurs financiers.

\section{Initiatives internationales ambivalentes}

\section{a) Le paper tracing}

L'explosion du trafic des stupéfiants et des techniques qui l'accompagnent, c'est-à-dire le blanchiment d'argent, a contraint les autorités judiciaires à adapter leurs stratégies d'investigation. Traditionnellement, après avoir reconstruit le 
crime et son modus operandi, ainsi qu'après avoir identifié l'auteur, l'enquêteur cherche à remonter au revenu du crime. Le trafic illicite accompli à échelle internationale par la criminalité organisée produit des revenus importants au point qu'il devient de plus en plus difficile de les cacher. Ces revenus sont constitués, en général, d'argent comptant, souvent en petites coupures. Selon la stratégie d'enquête actuelle, on cherche à localiser ces revenus dans le but de pouvoir identifier les coupables. II est dès lors nécessaire de suivre la trace de ces revenus figurant dans les documents des institutions bancaires et financières où ils ont été blanchis. II est évident que cette trace (paper trail) peut passer par les canaux clandestins destinés traditionnellement à cacher des capitaux sortis pour des raisons fiscales ou monétaires. La trace peut donc également passer par des comptes bancaires de sociétés ayant leur siège ou leur boîte à lettres dans un pays refuge-fiscal ou refuge-pénal ou par les comptes bancaires des responsables des filières qui se consacrent à la contrebande internationale de devises. C'est la raison pour laquelle on a invoqué encore récemment la nécessité d'assurer aux organes de police - au niveau d'Interpol par exemple - la collaboration d'experts-comptables.

\section{b) L'affaiblissement du secret bancaire}

La réalisation de la technique du paper tracing suppose pouvoir surmonter l'obstacle constitué par le secret bancaire, notamment dans les pays où la législation prévoit aujourd'hui encore des restrictions à l'accès aux informations et aux documents bancaires de la part des autorités judiciaires et de police.

Il est dès lors aisément compréhensible que l'on ait constamment cherché à assouplir la protection offerte par le secret bancaire. L'initiative la plus spectaculaire a été lancée par les Etats-Unis en 1983 à l'égard des pays des Caraïbes (Caribbean Basin Initiative - CBI). Selon cette législation nord-américaine, 27 Etats de cette région peuvent bénéficier de certains avantages économiques à condition de conclure avec les Etats-Unis un accord bilatéral relatif à un échange d'informations. En effet, ces Etats sont invités à des échanges d'informations dans le cadre de poursuites du trafic de drogue et autres crimes ou relatif à l'échange d'informations en matière fiscale. Les accords en question prévoient notamment l'échange d'informations au sujet d'actions au porteur et de comptes bancaires. De nombreuses initiatives visant l'assouplissement du secret bancaire ont aussi vu le jour au sein des organes de multiples organisations internationales.

\section{c) L'entraide judiciaire internationale facilitée}

La concentration de revenus d'origine criminelle en main d'une seule personne va la conduire à adopter des mesures pour échapper aux difficultés d'ordre fiscal. Si ses revenus ne sont pas déclarés, son train de vie disproportionné à 
l'égard de son activité professionnelle connue risque d'attirer les soupçons des autorités. Les responsables du crime organisé essaient dès lors de faire suivre la première phase de blanchiment des revenus d'origine criminelle d'une deuxième phase de recyclage à l'intérieur du circuit économique légal, de façon à pouvoir déclarer lesdits revenus au fisc. Cette deuxième phase comporte des opérations comptables que les autorités fiscales peuvent dévoiler. C'est ainsi qu'est née une "nouvelle" tactique d'investigation confiant aux autorités fiscales les enquêtes contre les personnes soupçonnées. Cette technique typiquement nord-américaine a même trouvé l'approbation du Conseil fédéral suisse, qui a déclaré :

"La punition de l'inobservation des dispositions légales relatives à l'imposition de revenus personnels est souvent le seul moyen pour déférer en justice les vrais responsables du crime organise." (Voir chiffre 31 du Message du Conseil fédéral $n^{\circledR} 12070$ du 28 août 1974 concernant le Traité avec les Etats-Unis d'Amérique).

Reconnaissant l'utilité de cette technique d'enquête, le Parlement suisse a approuvé des normes d'entraide facilitée en matière fiscale pour des poursuites américaines ouvertes contre les membres du crime organisé (art. 6 à 8 du Traité d'entraide entre la Suisse et les Etats-Unis). En effet, en orientant les enquêtes sur les infractions de caractère fiscal, il est possible de pénétrer "dans l'anonymat dont s'entourent les organisations criminelles et notamment leurs dirigeants, grâce à l'obligation du silence et du secret." Cette nouvelle technique d'investigation permet également de s'attaquer au problème posé par la division du travail à l'intérieur du crime organisé; les chefs de la hiérarchie ne sont liés que très indirectement aux crimes commis par l'organisation elle-même, et il est ainsi presque impossible d'obtenir des preuves de leur participation à des crimes déterminés. La législation italienne contre la mafia, adoptée en 1985, a suivi ce chemin. Les personnes ayant obtenu des gains en qualité de membres de la mafia sont tenues de par la loi de les déclarer au fisc. Le Parlement italien avait considéré que la preuve d'un manquement à cette obligation serait plus facile à apporter que la preuve de la commission d'un crime déterminé de la part des membres de la mafia. Cette législation a permis aux autorités judiciaires italiennes de confisquer des biens pour une valeur totale de mille cinq cent milliards de lires entre 1985 et 1988.

d) La coopération internationale directe entre autorités administratives et de police

Pour lutter contre la stratégie actuelle du crime organisé qui cherche à s'infiltrer dans le secteur bancaire et financier, les autorités des Etats-Unis proposent deux initiatives:

1) un projet d'extension à tous les pays du monde du système américain du reporting selon lequel toute opération cash d'une valeur dépassant les dix mille dollars doit être notifiée à l'autorité (Kerry Amendment de 1988); 
2) la multiplication des enquêtes masquées par des agents infiltrés dans le secteur financier.

Il est évident que la collaboration directe entre les autorités chargées de l'accès et de l'informatisation des données concernant toutes les transactions financières dans le réseau financier international, et celles chargées de conduire des enquêtes moyennant des infiltrés dans le secteur financier, réduira considérablement toutes les possibilités actuelles du contrôle judiciaire sur le transfert d'informations à caractère privé utilisées par le pays requérant dans le but de promouvoir des procédures fiscales ou monétaires.

\section{En guise de conclusion}

\section{Deux, trois, plusieurs chevaux de Troie}

Depuis des années la coopération internationale en matière purement fiscale s'enlise. Le sort de la fameuse Convention multilatérale européenne pour la coopération fiscale, élaborée par l'OCDE et déposée pour signature auprès du Conseil de l'Europe dès le 4 janvier 1988, en est un bon exemple. A ce jour, elle n'a pas encore réuni les cinq ratifications nécessaires à son entrée en vigueur et rien ne laisse prévoir que les gouvernements s'empresseront de la ratifier. Un autre exemple: la proposition d'un impôt à la source homogène pour tous les pays membres de la Communauté Européenne a été étouffée, au début de 1989, avant de pouvoir prendre forme. Par ailleurs, quelques Etats ont déjà manifesté leur préoccupation au sujet d'éventuels effets collatéraux préjudiciables pour la fiscalité nationale inhérents à la libéralisation du marché des capitaux prévue pour 1993. Officiellement - comme l'avait fait en avril $1989 \mathrm{M}$. Ciampi, gouverneur de la Banque d'Italie - on craint que la prochaine abolition des barrières douanières et des restrictions monétaires entraîne également une libéralisation des flux de capitaux d'origine criminelle. En réalité cependant, certaines administrations nationales considèrent le contrôle du transfert des devises comme un instrument de contrôle fiscal et se préoccupent par conséquent des effets de son abolition.

L'amélioration de la technique d'enquête du paper tracing transnational est un véritable cheval de Troie qui doit permettre de pénétrer dans les sanctuaires du blanchiment et de la gestion des capitaux d'origine criminelle par delà le secret bancaire. Mais ce cheval est accompagné d'un deuxième qui, lui, cherche à pénétrer dans les sanctuaires protégés par la discrétion du secret bancaire et qui abritent et gèrent des capitaux en fuite pour des raisons fiscales. II constitue un véritable cauchemar pour les banquiers des centres financiers dont la force ne repose que sur le secret bancaire. Les banquiers trouvent des alliés dans les milieux qui craignent un troisième cheval de Troie : celui qui est destiné à garantir l'instauration d'un contrôle politique. En effet, dans certains pays, des régions géographiques, des secteurs de l'administration publique et de l'économie pri- 
vée sont actuellement sous une certaine influence du crime organisé. A l'égard de pays qui vivent une période de troubles et d'incertitudes politiques, des administrations et des services étrangers pourraient être tentés d'utiliser les canaux de la coopération privilégiée prévus pour combattre le blanchiment d'argent dans le but d'acquérir une position de contrôle dans le pays concerné. Nul n'ignore que le trafic des stupéfiants peut également avoir une connotation politique, d'une part à cause de ses relations avec le trafic d'armes et d'autre part à cause de son pouvoir de corruption à l'égard des secteurs faibles de l'administration.

Jusqu'à présent, l'attitude des milieux politiques et économiques en Suisse s'est caractérisée par un maximum d'efficacité et de coopération accordées aux autorités judiciaires étrangères pour la répression de la criminalité de droit commun. A chaque fois que la pression étrangère a augmenté, des concessions dans ce secteur ont été faites dans le but de ménager le secteur touchant la coopération fiscale. Cette stratégie a été efficace jusqu'à présent. Même les centres financiers qui ont joué, à l'égard de la Suisse, la carte de la competition by laxity se sont résignés à la suivre. Ils ont adopté en 1989 des initiatives jusqu'alors surprenantes. En réalité, un nouveau tournant s'est effectué.

Après que maints juristes - diplomates, fonctionnaires, juges - ont livré avec succès un remarquable combat d'arrière-garde, le défi de l'adaptation et du renouvellement passe maintenant en mains des milieux bancaires et financiers. 


\section{Blbliographle}

\section{Première partle: criminalité et blanchissage d'argent}

\section{A. Références générales}

- President Commission, The cash connection, Washington, U.S. Government, Printing Office, 1986.

- National Commission Treadway, Report of the National Commission on fraudolent financial reporting, Washington, octobre 1987.

- Michael COMER, Corporate fraud, Londres, McGraw-Hill Book Ltd (UK), 1985.

- Ralph Blumenthal, Last days of the Sicilians, The FBI assault on the pizza connection, New York, Random House inc., 1988.

- Diane FRANCIS, Contrepreneurs, Toronto, Ed. Macmillan, 1988.

- Richard BLUM, Off-shore, Heaven Banks, Trust and Companies. The business of crime in the Euromarket, New York, Ed. Praeger, 1984.

- Donald MOFFITT, SWINDLED, Classic business frauds of the Seventies, New-York, 1976.

- Mafia, L'atto d'accusa di guidici di Palermo, Rome, Editori Riuniti, 1986.

- Sindona, Gli atti d'accusa dei giudici di Milano, Rome, Editori Riuniti, 1986.

- Relazione del Procuratore Generale per l'inaugurazione del anno Giudiziario 1989 , Milan, 14 1.89, Edizioni Tiemme, 1989.

- Vincente di Stefano, "Manipulazioni finanziarie e riciclaggio dei profitti illeciti" in: Gli investimenti della mafia, CEPIG, Latina, 1987 (pp. 195 et ss.)

- International Enforcement Law Reporter, Washington, D.C., 1989.

- McLure Charles, US Tax Laws ans Capital Flight from America latina, The University of Miami Inter American Law Review, No 2 Spring 1989.

- Malarek Victor, Merchants of Misery, Toronto,1984.

- Interpol, Colloque international sur la traite des êtres humains, Paris, 21-23 septembre 1988.

- Interpol, Deuxième colloque européen sur les activités frauduleuses et la criminalité économique, Paris, 14-15 février 1989.

- Interpol, Colloque européen sur le blanchiment d'argent, Paris, 18-20 avril 1989.

- Conseil de l'Europe, La criminalité des affaires, Strasbourg, 1981.

- Rudolf MULLER, Heinz BERND, WABNITZ, Wirtschaftskriminalität, München, Verlag C.H. Beck, 1986.

- "Fight to the death. Colombia takes on the lords of cocaine", Time, 4.9.1989, p. 12 et ss.

- Groupe d'étude du G7, Rapport concernant la lutte internationale contre le blanchissage d'argent, 1989, (ce groupe a été institué par décision prise au sommet des représentants des sept pays les plus industrialisés du monde tenu à Toronto en juin 1988. Le rapport concluait avec la proposition de constitution d'un fonds international dans le but de mettre à disposition les moyens nécessaires pour financer des opérations analogues à celles conduites depuis des années par la Drug Enforcement Agency (DEA). A titre de démonstration de la validité de cette technique investigative, les autorités USA citent la découverte d'un réseau de blanchisseurs d'argent à l'intérieur de la Banque de Crédit et de Commerce International, un groupe bancaire multinational avec siège au Luxembourg. L'autorité judiciaire de Tampa (Floride) a 
découvert des faits sur la base desquels ont été décernés, en 1988, des mandats d'arrestation contre une quarantaine de personnes dont huit appartenant aux cadres de ce groupe bancaire.

\section{B. Casuistique suisse}

- Rapport de la commission parlementaire d'enquête du 22.11.1989 (affaire Kopp) Berne, 1989

- Conseil fédéral, Message concernant le Traité avec les Etats-Unis d'Amérique pour l'entraide judiciaire en matière pénale du 28 août 1974 (p. 6).

- Hoets, Zwart, Swiss Bank Secrecy and the Marcos Affair, New York Law School Journal of International and Comparative Law, Vol. 9, № 1, 1988

- Conseil fédéral, Message concernant la modification du code pénal suisse. Législation sur le blanchissage d'argent et le défaut de vigilance en matière d'opérations financières du 12 juin 1989 (p. 6).

- THURSTON, Clarke, John TIGUE Jr, Dirty Money Swiss Banks, the Mafia Money Laundering and White Collar Crime ${ }_{\perp}$ New York, Ed. Simon \& Schuster, 1975.

- Nicolas FAITH, Safety in number. The mysterious world of swiss banking, Londres, 1982.

- T. R. FEHRENBACH, The Gnomes of Zurich, Londres, Ed. Leslie Frewin, 1966.

- Commission fédérale des banques, "Avoirs de Marcos", dans: Rapport de gestion 1987 (p. 156).

- Paolo BERNASCONI, Finanzunterwelt, Zurich, Orell Füssli Verlag, 1988.

- Commission fédérale des banques, "Blanchissage d'argent", dans : Rapport de gestion 1987 (p. 158) ainsi que dans : Rapport de gestion 1988 (p. 152).

- Commission fédérale des banques, "Untersuchung der Eidgenössischen Bankenkommission über das Verhalten der Grossbanken in Sache Magharian - Geldwäscherei Lebanon connection", conférence de presse de la Commission fédérale des banques du 11 avril 1989.

- Maurice AUBERT, Le secret bancaire, la Suisse et les autres Etats, Genève, 1975 (p. 35 : L'or du Vietnam du Sud et du Cambodge).

- Conseil fédéral, Réponses aux interpellations du Conseil national en matière de trafic illicite d'armes depuis la Suisse (No 87. 446, 87.405, 86.760), Bulletin sténographique du Conseil national du 18 septembre 1979, p. 964 et ss.

- Conseil fédéral, Réponse écrite à une interpellations du Conseil national du 29.11.1979 ( No 79.547).

- Conseil fédéral, Réponse écrite 2 mars 1987 à une interpellation du Conseil national du 8 octobre 1986 (No 86.928 Statistique des cas de blanchiment d'argent)

- Conseil fédéral, Réponses aux interpellations présentées au Conseil national concernant les avoirs de Marcos en Suisse (No 86.401, 86.482, 86.913) cf. Bulletin sténographique du Conseil national pp. 421-434, 1987.

- Conseil fédéral, Réponse à l'interpellation du Conseil national du 19 décembre 1986 concernant les banques suisses et Irangate (No 86.811 Rapport du 2 mars 1987).

- Office fédéral de Justice, Situationsanalyse der Interdepartementalen Arbeitsgruppe Frauen aus Dritten Welt, juin 1988, Berne.

- Pierre KELLER, "L'accord avec les Etats-Unis concernant les opérations d'initiés", dans : Ein Blick in die schweizerische Aussenpolitik, Festschrift,Berne; 1985, (casuistique concernant les opérations d'initiés entre la Suisse et les Etats-Unis). 
- Peter KLAUSER, "Das schweizerisches Bankgeheimnis und seine internazionale Tragweite", Wirtschaft und Rechts, Sonderheft, Zurich, 1977.

- Tribunal fédéral, Jugements publiés concernant des cas liés au blanchissage d'argent en Suisse : transfert à Genève du produit d'une escroquerie commise à Taïwan (RO 109 IV 1) transfert à Zurich d'une escroquerie commise à New York (RO. 99 IV 121); transfert des avoirs de Licio Gelli à Genève (RO. 112 I b 632); transfert à Lugano du produit de la corruption dans le scandalo dei petroli de Turin (ct. B. Trinkler, op. cit., Steuer Revue, 4/1985)

- Paolo BERNASCONI, "Le recyclage de l'argent d'origine criminelle", Revue internationale de criminologie et de police technique, Genève, 1981, pp. 403 et ss.; (casuistique concernant le blanchissage en Suisse de rançons versées par les familles des personnes enlevées en Italie).

- Irma WEISS, "Die Einziehung in der Schweiz liegender Vermögen aus ausländischen Drogenhandel", Revue pénale suisse 102, 1985, pp. 203 et ss.

- Bruno TRINKLER, "Die Behandlung von Rechtshilfegesuche in fiskalischen Strafsachen im Kanton Zurich nach dem IRSG", Stever Revue 4/1985, p. 193 et ss.

- Bruno TRINKLER, "Aus der Praxis des Kantons Zurich zur internationalen Rechtshilfe", Revue pénale suisse, 104, 1987, pp. 208 et ss.

- Association Genevoise du droit des affaires, Colloque, La convention de diligence et les nouvelles normes pénale suisse contre le blanchiment d'argent, Actes du Colloque du 13.12.1989, Genève.

C. Reportages de presse concernant la casuistique suisse :

- "Marc Rich", NZZ, No 195, 199, 1983.

- "Scoperti in capitali di Ciancimino ex-Sindaco di Palermo nelle banche svizzere", Corriere del Ticino, 8 mai 1987.

- "Affaire banquier suédois avec siège dans le canton de Zoug", Dagens Nyheter, 29 avril 1987.

- "Affaire de la vente des armes suédoises (Bofors) à l'Inde", Swedish Press Digest, 14 mai 1987.

- Chitra SUBRAMANIAN, "Ventes d'armes suédoises à l'Inde. Pattes graissées en Suisse", Le Courrier, 15 juin 1987.

- Treasure hunt, "Did Soekarno bury billions in Barclays and the Swiss Bank ?", The Wallstreet Journal 29 février 1988.

- "Armée rouge japonaise, comptes suisses pour terroristes", Tribune de Genève. 18 avril 1988.

- "Fonds Duvalier à Genève", Tribune de Genève, 16 février 1989

- "Die schweizer Verstrickung in die Affaire Lucona", NZZ, 20 avril 1989.

- "Die Marcos Gelder auf schweizer Konti", NZZ, 30 mai 1989.

Deuxième partie: Elusion, évasion et fraude fiscale, fuite de capitaux, paradis fiscaux

- Conseil de l'Europe, Convention concernant l'assistance administrative mutuelle en matière fiscale, Strasbourg, 25 janvier 1988. 
- Comité des affaires fiscales de l'OCDE, L'évasion et la fraude fiscales internationales, Paris, 1987.

- John WILLIAMSON, Donald LESSARD, "Capital flight : The problem and policy responses", In : Institute for International Economics, novembre 1987.

- Robin-Thomas NAYLOR, Hot Money, New York, Ed. Simon \& Schuster, 1987.

- André BEAUCHAMP, Guide mondial des paradis fiscaux, Paris, Grasset, 1987.

- Walter INGO, Secret Money, The world of international financial secrecy, Londres, Ed. George Allen Unwinn, 1987.

- Collectif, Schuldenkrieg und CH-Finanzkapital IWF, Entwicklungspolitik und Solidaritätsbewegung-Analysen, Kontroverse, Widerstand, dans: Widerspruch, Sonderband 2., Zurich, avril 1989.

- Olivier STANLEY, Giles CLARKE, Offshore Tax Planning, Butterworth, London, 1988.

- Morgan \& Morgan Corporation Services S.A., Formation Management and Administration of Companies British Vierges Islands, Genève, 1989 (ct. aussi PANAZUR Corporate \& Shipping Services Inc, Zurich, Panama Gesellschaften, Zurich, 1988.

- "Taxing Luxemburg ", dans: Offshore Adviser, Financial Times publication, 23 mars 1989, p. 9.

- "The Battle of offshore Britain" in: Offshore Investment, International Magazine for Money, Business and Property offshore, Vol. 2 No 1.

- Rudolf BAECHTOLD, Eine Adresse in Liechtenstein. Finanzdrehscheibe und Steuer Paradies, Wiesbaden, 1979.

- Heinz FROMMELT, Das liechtensteinische Bankgeheimnis, Zurich, Schulthess Polygraphischer Verlag, 1989.

- "Ein Fast sauberes Ländli", Round Table, Bilanz, 9, 1986, p. 97.

- "Steuer und Bankenrechtlische Fragen in Liechtenstein", Referate des Liechtenstein Seminars du 14 novembre 1988, Vaduz, 1988.

- CCH International, International Offshore Financial Centres, New York, Horwath \& Horwath international, 1988.

- Crime and Secrecy : the use of offshore banks and companies, Staff Study made by Permanent Subcommittee on Investigations of the Committee on governmental affairs, US Senate 1983/1985, Washington, U.S. government printing office.

- Conseil fédéral, Réponses aux interpellations du Conseil national concernant les banques suisses et le recours à des établissements du Liechtenstein et du Panama (Bulletin sténographique du Conseil nationalı. pp. 944-950, pp. 1693-1697, 1978).

- Conseil fédéral, Réponse à l'interpellation du Conseil national du 3 juin 1985 concernant la recommandation de l'OCDE concernant le secret bancaire (Bulletin du Conseil national, pp. 1070-1075).

- Conseil fédéral, Gouvernement des Etats-Unis, Memorandum of understanding du 10 octobre 1987.

- Association suisse des banquiers, Convention relative à l'obligation de diligence des banques du 1er juillet 1987 (ct. à ce sujet "Die Neue Vereinbarung über die Sorgfaltspflichten der Banken" dans Wirtschaft und Recht, Sonderheft, 1987).

- Thomas NAYLOR, "Money laudering. A political and macroeconomic perspective", conférence tenue au colloque de Florence le 20 mai 1989, La drogue en Europe, organisé par FeDro, Florence, (polycopié). 


\section{Trolsième partie: Léglslation concernant le blanchlssage d'argent}

Etats-Unis d'Amérique:

- Laundering of Monetary Instruments (18 U.S.C.) 1956-1957.

- Omnibus Drug Legislation (p. 4702).

- Restrictions on laundering of United States currency.

- Bank Records and foreign transactions Act, 1970 (appelé aussi Bank Secrecy Act).

Royaume-Unis:

- Drug Trafficking Offences Act, 1986 (Section XXXII).

- Prevention of Terrorism Act, 1989 (Section IV).

\section{France:}

Loi N. 87 - 1157 du 31.12 .1987 relative à la lutte contre le trafic de stupéfiants et modifiant certaines dispositions du Code pénal.

Italie:

- Legge 13 septembre 1982 No 646 (législation contre les organisations de la mafia).

Panama:

Ley N. 23 du 23 décembre 1986 pour la révision partielle du Code pénal.

OIPC - Interpol:

Loi type destinée à faciliter le recueil des éléments de preuve nécessaires aux enquêtes et procédures pénales ainsi qu'à la confiscation du produit des activités criminelles. 